\title{
Publisher's Note: Controlling the Goos-Hänchen and Imbert-Fedorov shifts via pump and driving fields [Phys. Rev. A 93, 013821 (2016)]
}

\author{
Saeed Asiri, Jingping Xu, M. Al-Amri, and M. Suhail Zubairy \\ (Received 27 January 2016; published 9 February 2016)
}

DOI: 10.1103/PhysRevA.93.029901

This paper was published online on 13 January 2016 with an omission from the Acknowledgments. The last sentence of the Acknowledgments should read as "The research of J.X. is supported by the National Natural Science Foundation of China (Grants No. 11274242 and No. 11574229), and Shanghai Science and Technology Committee for Grant No. 15XD1503700." The Acknowledgments have been corrected as of 27 January 2016. The Acknowledgments are correct in the printed version of the journal. 